\title{
A short proof and generalization of Lagrange's theorem on continued fractions
}

\author{
Sam Northshield
}

\begin{abstract}
We present a short new proof that the continued fraction of a quadratic irrational eventually repeats. The proof easily generalizes; we construct a large class of functions which, when iterated, must eventually repeat when starting with a quadratic irrational.
\end{abstract}

\section{Introduction.}

A quadratic irrational is an irrational root of a quadratic polynomial with integer coefficients. Lagrange's theorem on continued fractions - that any positive quadratic irrational has an eventually repeating continued fraction - has many proofs. Perhaps the most common proof is one by Charves which can be found in a book by Hardy and Wright [1, Theorem 177]. Many other proofs exist of course; a particularly short proof appears in the book by Hensley [2, p. 9] and a more general result appears in a recent paper by Panti [3]. We present a short new proof which leads to a new generalization.

\section{Lagrange's Theorem.}

Let $\left\langle a_{0}, a_{1}, a_{2}, \ldots\right\rangle:=a_{0}+1 /\left(a_{1}+1 /\left(a_{2}+1 / \ldots\right)\right)$ where each $a_{i}$ is an integer and, for some $N, a_{i}=0$ for $i<N$ and $a_{i}>0$ for $i \geq N$. This, of course, is not quite the standard notation for continued fractions. In particular, there are infinitely many representations for a given number. However, if $\left\langle 0,0, \ldots 0, a_{N}, a_{N+1} \ldots\right\rangle=$ $\left\langle 0,0, \ldots 0, b_{M}, b_{M+1} \ldots\right\rangle$ where $a_{N}>0$ and $b_{M}>0$, then $M-N$ is even and $b_{M+k}=a_{N+k}$ for all $k$. Define a function on positive real numbers by

$$
f(x):= \begin{cases}x-1 & \text { if } x \geq 1 \\ x /(1-x) & \text { if } x<1 .\end{cases}
$$

Note that $\left\langle 0,0, \ldots 0, a_{N}, a_{N+1} \ldots\right\rangle>1$ if and only if $N$, the number of zeros, is even and, in general,

$$
f\left(\left\langle 0,0, \ldots 0, a_{N}, a_{N+1} \ldots\right\rangle\right)=\left\langle 0,0, \ldots, 0, a_{N}-1, a_{N+1}, \ldots\right\rangle .
$$


Iteration of $f$ then chips away at the leftmost nonzero integer in $\left\langle a_{0}, a_{1}, a_{2}, \ldots\right\rangle$, reducing it by one in each step.

Suppose $x$ is a positive quadratic irrational. Then $x$ is irrational and there exist integers $a, b, c$ such that $a x^{2}+b x+c=0$. We use the notation $x \in[a, b, c]$ for this. It is then easy to verify that

$$
a(x-1)^{2}+(2 a+b)(x-1)+(a+b+c)=a x^{2}+b x+c=0
$$

and

$$
(a+b+c) x^{2}+(b+2 c) x(1-x)+c(1-x)^{2}=a x^{2}+b x+c=0,
$$

and thus

$$
f(x) \in[a, 2 a+b, a+b+c] \text { or } f(x) \in[a+b+c, b+2 c, c] .
$$

Let $x_{1}:=x$ and, for $n \geq 1, x_{n+1}:=f\left(x_{n}\right)$. Then $\left(x_{n}\right)$ is a sequence of quadratic irrationals and so determines an infinite sequence of triples: $x_{n} \in\left[s_{n}, t_{n}, u_{n}\right]$ where we may assume, without loss of generality, that $s_{n}>0$ (since $y \in[s, t, u]$ if and only if $y \in[-s,-t,-u])$. Since

$$
(2 a+b)^{2}-4 a(a+b+c)=b^{2}-4 a c=(b+2 c)^{2}-4(a+b+c) c,
$$

we see that $t_{n}^{2}-4 s_{n} u_{n}$ is independent of $n$.

If only finitely many of the triples $\left[s_{n}, t_{n}, u_{n}\right]$ have $u_{n}<0$, then from some point on, $s_{n}, u_{n}>0$ and, consequently, $t_{n}<0$ (because $x_{n}>0$ ). This is impossible since $\left(s_{n}-t_{n}+u_{n}\right)$ would then be strictly decreasing and nonnegative. Therefore, $s_{n} u_{n}<0$ infinitely often and, since $t_{n}^{2}-4 s_{n} u_{n}$ is constant, there must be a triple which appears three times in the sequence $\left(\left[s_{n}, t_{n}, u_{n}\right]\right)$. Hence $x_{n}=x_{m}$ for some $m$ and $n$ satisfying $m>n$. If $x=\left\langle a_{0}, a_{1}, a_{2}, \ldots\right\rangle$ then $x_{n}$ is of the form $\left\langle 0, \ldots, 0, b, a_{i}, a_{i+1}, \ldots\right\rangle$ and $x_{m}$ is of the form $\left\langle 0, \ldots, 0, c, a_{j}, a_{j+1}, \ldots\right\rangle$ where $b>0, c>0$, and, necessarily, $j>i$. Since these are equal, the difference $j-i$ is positive and even and so $b=c$ and, for all $k, a_{j+k}=a_{i+k}$. That is, the sequence $a_{k}$ is eventually periodic and we have Lagrange's theorem:

Theorem 1. If $x$ is a positive quadratic irrational then its continued fraction is eventually periodic.

\section{Generalizations.}

There are only three facts about $f$ necessary so that if $x$ is a quadratic irrational then $x_{n}$ eventually repeats. They are that $f$ takes positive numbers to positive numbers, that for any of the corresponding triples $\left[s_{n}, t_{n}, u_{n}\right], t_{n}^{2}-4 s_{n} u_{n}$ is independent of $n$, and that whenever $s_{n} u_{n}>0$ and $s_{n+1} u_{n+1}>0,\left|s_{n+1}\right|+$ $\left|t_{n+1}\right|+\left|u_{n+1}\right| \leq\left|s_{n}\right|+\left|t_{n}\right|+\left|u_{n}\right|$.

We say that a function is regular if it is a fractional linear transformation $(a x+b) /(c x+d)$ where $a, b, c, d$ are integers satisfying $|a d-b c|=1,(a-b)(d-c)>$ 0 , and there exists $t>0$ such that $(a t+b) /(c t+d)>0$. (This last condition, although made redundant by the hypotheses of the next two theorems, will be useful later.) We then have: 
Theorem 2. Let $f:(0, \infty) \rightarrow(0, \infty)$ be any function which is piecewise regular. If $x$ is a positive quadratic irrational then the iterates of $f$, starting at $x$, eventually repeat.

Proof. It is not hard to verify that for any $s, t, u, a, b, c, d$, if $S=d^{2} s-c d t+c^{2} u$, $T=-2 b d s+(a d+b c) t-2 a c u$, and $U=b^{2} s-a b t+a^{2} u$, then

$$
t^{2}-4 s u=(a d-b c)^{2}\left(T^{2}-4 S U\right)
$$

and

$$
S(a x+b)^{2}+T(a x+b)(c x+d)+U(c x+d)^{2}=(a d-b c)^{2}\left(s x^{2}+t x+u\right) .
$$

Suppose $x$ is a quadratic irrational, so that there exist integers $s, t, u$ such that $s x^{2}+t x+u=0$. Then for $S, T$, and $U$ as defined above,

$$
S\left(\frac{a x+b}{c x+d}\right)^{2}+T\left(\frac{a x+b}{c x+d}\right)+U=0 .
$$

Let $x_{1}:=x$ and, for $n \geq 1, x_{n+1}=f\left(x_{n}\right)$. By the hypothesis of the theorem, if $x_{n} \in\left[s_{n}, t_{n}, u_{n}\right]$, then $x_{n+1} \in\left[s_{n+1}, t_{n+1}, u_{n+1}\right]$ where $t_{n+1}^{2}-4 s_{n+1} u_{n+1}=$ $t_{n}^{2}-4 s_{n} u_{n}$. Since there are only finitely many triples $[a, b, c]$ where $b^{2}-4 a c$ is bounded and $a c<0$, either there exist $i, j, k$ such that $i<j<k$ and $\left[s_{i}, t_{i}, u_{i}\right]=\left[s_{j}, t_{j}, u_{j}\right]=\left[s_{k}, t_{k}, u_{k}\right]$ (hence, at least two of $x_{i}, x_{j}, x_{k}$ agree) or $s_{n} u_{n}>0$ for all sufficiently large $n$.

Suppose $x \in[s, t, u]$ and $f(x)=(a x+b) /(c x+d)$. Let $S=d^{2} s-c d t+c^{2} u$, $T=-2 b d s+(a d+b c) t-2 a c u$, and $U=b^{2} s-a b t+a^{2} u$ so that $f(x) \in[S, T, U]$. It is not hard to verify that, since $|a d-b c|=1$,

$$
s-t+u=(a-b)^{2} S-(a-b)(d-c) T+(c-d)^{2} U .
$$

Now suppose that $S U, s u>0$. Then since $x$ and $f(x)$ are positive, $t$ must have the opposite sign from $s$ and $u$, and $T$ must have the opposite sign from $S$ and $U$. Also, since $(a-b)(d-c) \geq 1$, we must have $(a-b)^{2} \geq 1$ and $(c-d)^{2} \geq 1$. Therefore,

$$
\begin{array}{r}
|s|+|t|+|u|=|s-t+u|=\left|(a-b)^{2} S-(a-b)(d-c) T+(c-d)^{2} U\right| \\
=(a-b)^{2}|S|+(a-b)(d-c)|T|+(c-d)^{2}|U| \geq|S|+|T|+|U| .
\end{array}
$$

Since there are only finitely many triples $[a, b, c]$ where $|a|+|b|+|c|$ is bounded, there must exist $i, j, k$ such that $i<j<k$ and $\left[s_{i}, t_{i}, u_{i}\right]=\left[s_{j}, t_{j}, u_{j}\right]=$ $\left[s_{k}, t_{k}, u_{k}\right]$. Hence, at least two of $x_{i}, x_{j}, x_{k}$ agree and the result follows.

Example 1. If $f(x)=\{1 / x\}$ (where $\{x\}$ denotes the fractional part of $x$; this $f$ is usually called the Gauss map) and $x$ is a positive quadratic irrational then the iterates of $f$ eventually repeat.

Example 2. If $f(x)=\{x\} /(1-\{x\})$ and $x$ is a positive quadratic irrational then the iterates of $f$ eventually repeat. 
We may extend further; the proof of the following theorem is essentially contained in that of Theorem 2 and is left to the reader.

Theorem 3. Let $f_{1}, f_{2}, \ldots$ be any sequence of regular functions. Given $x$, define a sequence recursively by $x_{1}:=x$ and, for $n \geq 1, x_{n+1}=f_{n}\left(x_{n}\right)$. If $x$ is a positive quadratic irrational and $x_{n}>0$ for all $n$, then there exist distinct $j, k$ such that $x_{j}=x_{k}$.

Example 3. For any $n$, choose one of the two functions $\{1 / x\}$ or $\{x\} /(1-$ $\{x\})$ at random and so form a random sequence $f_{n}$. For any positive quadratic irrational $x$, the sequence $\left(x_{n}\right)$ defined by $x_{1}:=x$ and $x_{n+1}:=f_{n}\left(x_{n}\right)$ satisfies $x_{j}=x_{k}$ for some pair of distinct integers $j, k$.

\section{Understanding Regular Functions.}

Recall $P G L_{2}(\mathbb{Z})$ can be taken to be the group of linear fractional transformations $(a x+b) /(c x+d)$ where $a, b, c, d \in \mathbb{Z}$ and $a d-b c= \pm 1$. A function $g(x)$ is then regular if it is an element of $P G L_{2}(\mathbb{Z})$ such that $g(-1)<0$ and there exists $t>0$ such that $g(t)>0$. It turns out, by a careful consideration of several cases, that if $g$ is regular then $g(s)<0$ for all $s<0$. Since $g$ takes on all values except possibly $\lim _{x \rightarrow-\infty} g(x)$, which is nonpositive, the range of $g$ must contain all positive numbers. It follows that the set of regular functions is closed under composition.

We may then classify all regular functions. First, $g$ is regular with determinant -1 if and only if $1 / g$ is regular with determinant 1 ; it is then enough to classify regular functions in $S L_{2}(\mathbb{Z})$. Given $a, b$ positive and relatively prime, let $a^{\prime}:=a^{-1}(\bmod b)$ and $b^{\prime}:=b^{-1}(\bmod a)$ (e.g., $a^{\prime}$ is the unique number between 1 and $b$ satisfying $\left.a a^{\prime} \equiv 1(\bmod b)\right)$ and define

$$
g_{a / b}(x):=\frac{a^{\prime} x+b^{\prime}-a}{\left(a^{\prime}-b\right) x+b^{\prime}} .
$$

It is not hard to show that $a a^{\prime}+b b^{\prime}=a b+1$ and that $g_{a / b}$ is regular and in $S L_{2}(\mathbb{Z})$. Conversely, every regular function $g$ in $S L_{2}(\mathbb{Z})$ is of that form! To see this, note that $g(x)=1$ has a positive solution since $g(s)<0$ for all $s<0$. Hence $g^{-1}(1)=a / b$ for some positive relatively prime $a$ and $b$. Suppose $g(x)=(s x+t) /(u x+v)$. Since $g(a / b)=1$, there exists $c$ such that $s a+t b=c=u a+v b$ and thus $u=s-b k$ and $v=t+a k$ for some $k$. By multiplying all of $s, t, u$, and $v$ by -1 if necessary, we may assume without loss of generality that $c>0$. Since $g \in S L_{2}(\mathbb{Z}),(a s+b t) k=s v-t u=1$ and thus $a s+b t=1, k=1, u=s-b$, and $v=t+a$. Since $g(-1)<0$, $(s-t)(t+a-s+b) \geq 1$ and so $0<s-t<a+b$. Since there is a unique pair $s, t$ such that $a s+b t=1$ and $0<s-t<a+b$, it follows that $s=a^{\prime}, t=b^{\prime}-a$, and the function $g$ must coincide with $g_{a / b}$.

We can go further. Suppose $a, b$ are positive integers with $a>b$. By the easily verified facts that $(a-b)^{-1}(\bmod b)=a^{-1}(\bmod b)$ and $b^{-1}(\bmod a-$ 
$b)=a^{-1}(\bmod b)+b^{-1}(\bmod a)-b$, it follows that $g_{(a-b) / b}(x-1)=g_{a / b}(x)$. Equivalently, for $r>1$,

$$
g_{r-1}(x-1)=g_{r}(x) .
$$

Since for positive rational $r, g_{1 / r}(1 / x)=1 / g_{r}(x)$, it follows that for $r \in(0,1)$,

$$
g_{r /(1-r)}(x /(1-x))=g_{r}(x) .
$$

Letting $f$ be defined as in Section 2 and given a positive rational $r$, there exists $n$ such that the $n$-fold iterate $f \circ f \circ \cdots \circ f(r)=1$. Hence there exists a sequence of functions $h_{1}, h_{2}, \ldots, h_{n}$ such that each $h_{i}(x)$ is either $x-1$ or $x /(1-x)$ and $H:=h_{n} \circ \cdots \circ h_{1}$ satisfies $H(r)=1$ and therefore

$$
g_{r}(x)=g_{H(r)}(H(x))=g_{1}(H(x))=H(x) .
$$

Hence every $g_{r}$ is a composition of the functions $x-1$ and $x /(1-x)$ and thus every regular function is a composition of the functions $x-1$ and $1 / x$. Since $x-1$ and $1 / x$ are regular and the set of regular functions is closed under composition, we see that the set of regular functions is the monoid generated by $x-1$ and $1 / x$.

This leads to a characterization of quadratic irrationals. Note that if $x=$ $\left\langle a_{0}, a_{1}, a_{2}, \ldots\right\rangle$, then $1 / x=\left\langle 0, a_{0}, a_{1}, a_{2}, \ldots\right\rangle$. Let

$$
S_{t}:=\{g(t): g \text { is regular and } g(t)>0\} .
$$

Since every $g$ is a composition of $x-1$ and $1 / x$, it follows that if $t=\left\langle a_{0}, a_{1}, a_{2}, \ldots\right\rangle$ then any element in $S_{t}$ is of the form $\left\langle 0, \ldots, 0, b, a_{n}, a_{n+1}, \ldots\right\rangle$, where $b \leq a_{n-1}$, and so $S_{t}$ is finite if and only if the sequence $\left(a_{n}\right)$ eventually repeats.

Theorem 4. A positive irrational number $t$ is a quadratic irrational if and only if $S_{t}$ is finite.

The regular functions in $S L_{2}(\mathbb{Z})$ can be parametrized by the positive rational numbers. This leads to an interesting associative (but not commutative) binary operation $*$ with identity 1 defined by $g_{r} \circ g_{s}=g_{r * s}$. Since $r * s=\left(g_{r} \circ g_{s}\right)^{-1}(1)$, one may write out $a / b * c / d$ explicitly:

$$
\frac{a}{b} * \frac{c}{d}=\frac{b c+(a-b) d^{\prime}}{a d+(b-a) c^{\prime}},
$$

where $c^{\prime}=c^{-1}(\bmod d)$ and $d^{\prime}=d^{-1}(\bmod c)$.

\section{References}

[1] G. H. Hardy and E. M. Wright, An Introduction to the Theory of Numbers, 5th ed., Oxford University Press, New York, 1979.

[2] D. Hensley, Continued Fractions, World Scientific, Hackensack, NJ, 2006.

[3] G. Panti, A general Lagrange theorem, this Monthly 116 (2009) 70-74.

Department of Mathematics, SUNY, Plattsburgh, NY 12901

northssw@plattsburgh.edu 